\title{
An Enhanced Spectrum Allocation Algorithm for Secondary Users in Cognitive Radio Networks
}

Sheenu Agarwal ( $\nabla$ sheenuagarwal001@gmail.com )

Birla Institute of Applied Sciences

\section{Sandip Vijay}

Shivalik College of Engineering

\section{Ashish Bagwari}

Women's Institute of Technology

\section{Research Article}

Keywords: Channel prediction, Channel sensing, Cognitive radio, Enhanced bee colony algorithm, Spectrum usage efficiency.

Posted Date: June 2nd, 2021

DOI: https://doi.org/10.21203/rs.3.rs-381522/v1

License: (c) (1) This work is licensed under a Creative Commons Attribution 4.0 International License. Read Full License 


\title{
An Enhanced Spectrum Allocation Algorithm for Secondary Users in
} Cognitive Radio Networks

\author{
${ }^{1}$ Sheenu Agarwal \\ Assistant Professor, Department of Electronics and Communication Engineering, Birla Institute \\ of Applied Sciences, Bhimtal \\ ${ }^{2}$ Dr. Sandip Vijay \\ Professor, Department of Electronics and Communication Engineering, Shivalik College of \\ Engineering, Dehradun \\ ${ }^{3}$ Dr. Ashish Bagwari \\ Associate Professor, Department of Electronics and Communication Engineering, Women's \\ Institute of Technology, Dehradun
}

\section{Corresponding Author:}

Sheenu Agarwal,

Birla Institute of Applied Sciences, Bhimtal

Email Id- sheenuagarwal001@gmail.com 


\begin{abstract}
:
Wireless communication is a vast field in order to do researches on transmitter sections, wireless mediums, receiver sections, repeaters as well as different antennas. The spectrum used for wireless communication is limited and the number of users is increasing day by day. To use the available spectrum efficiently cognitive radio were introduced in which different allocation schemes were proposed by the researchers. In this paper an enhanced artificial bee colony algorithm is proposed for predicting and sensing the channel of primary user which results in the efficient usage of the available spectrum via secondary users, the enhanced artificial bee colony algorithm first predict the channel allocated to primary user, thereafter it performs sensing of the channel due to which the false alarm probability reduced and overall throughput of the network increases. The proposed algorithm overcomes the drawbacks of modified traditional methodologies which were used only for sensing a channel and shows $11.8 \%$ more efficiency as compared to binary artificial bee colony algorithm. In future, the method for predicting the channel's availability can be enhanced in order to maximize the probability of channel allocation to the secondary user.
\end{abstract}

\title{
Keywords:
}

Channel prediction, Channel sensing, Cognitive radio, Enhanced bee colony algorithm, Spectrum usage efficiency.

\section{DECLARATIONS:}

\section{Funding:}

No funding was received for this work.

\section{Conflicts of interest/Competing interests:}

We wish to confirm that there are no known conflicts of interest associated with this publication and there has been no significant financial support for this work that could have influenced its outcome.

\section{Availability of data and material:}

Data openly available in a public repository that issues datasets with DOIs and Data derived from public domain resources.

\section{Code Availability:}

NA

\section{Author's Contribution:}

\section{NA}




\section{Acknowledgement:}

Authors acknowledge the immense help received from the scholars whose articles are cited and included in references of this manuscript. The authors are also grateful to authors/ editors/ publishers of all those articles, journals and books from where the literature for this article has been reviewed and discussed 


\section{INTRODUCTION}

Wireless communication [1] is evolving continuously since decades, it becomes an essential part of daily life from a television to the cell phones. The communication taken place between two entities via a wireless medium in order to provide a point to point or broadcast connection in between is called as wireless communication. During evolution of wireless communication some systems were grown and some got disappeared, the most flourished example of a wireless communication system is mobile communication [2] which connects so many users together. Wireless communication is reliable and cost effective as compared to the wired communication. Wireless communication is vastly used method of communication now a day. There are so many advantages of wireless communication like it is cost effective and easy to install, the data transferring can be done at high speed, it provides mobility to the user so that the user can use wireless medium at any place and the wireless communication eliminate the complexity of the network of cables. Other than its advantages wireless communication has some factors which affect the quality of the communication over the wireless medium. To explain the effect of these factors path loss model [3] is discussed.

There are so many obstacles like huge infrastructures, hills, dense forests etc. which affect the power of transmitted signal because it gets fade or scattered [4] after colliding with these structures. The signal can also be scattered by moving vehicles like trains, cars etc. To calculate the effect of these factors on the power of the received signals at the receiver, some parameters are considered.

$$
P_{r}=P_{t}\left[a\left(\frac{d_{o}}{d}\right)^{v} s l\right]
$$

Where,

- $P_{r}$ is power of the received signal at the receiver,

- $\mathrm{P}_{\mathrm{t}}$ is the power of the transmitted signal from the transmitter,

- $\mathrm{s}$ is the small scale fading factor,

- 1 is the large scale fading factor,

- $\mathrm{d}$ is the path length,

- $\mathrm{d}_{\mathrm{o}}$ is the reference distance,

- $\quad \mathrm{v}$ is path loss exponent and $\mathrm{a}$ is an adjustable parameter depends on physical factors.

Small scale fading: It occurs when the transmitted signal is scattered and received at the receiver via different paths or say multipath. It usually occurs when a user change its location rapidly over a short distance.

Large scale fading: It occurs when the user changes its location over a large distance. This type fading usually occurs due to scattering of the signal from the obstacles like buildings, moving vehicles etc.

Wireless communication uses radio frequency spectrum as a medium and the spectrum of limited bandwidth are assigned to different modes of communication, so there is a limitation in aspects of using these bandwidths due to which there exist a need to develop the techniques for optimizing the use of available bandwidth in the spectrum. To optimize the use of available 
bandwidth of the spectrum many techniques were introduced like time hopping techniques, frequency hopping techniques in order to provide resistance to the interference and eavesdropping. These techniques are able to perform for increasing the efficiency but are not so effective in order to utilize the whole bandwidth of the spectrum assigned to the user. The spectrum is used via all users whether they are licensed or not. The bandwidth is left without allotting the channels to some users which is a drawback of these techniques that the whole bandwidth is not utilized.

Therefore, to use the spectrum intelligently cognitive radio technology [5] is developed. Frequency spectrums are licensed but are not fully used by licensed users. The users are divided in two categories i.e. users which are licensed to use the spectrum are called as primary users and the users which are not licensed use cognitive radio technology for using that spectrum called as secondary users. Cognitive radio technology allows secondary users to use the spectrum when it is vacant or unused via primary users without affecting the frequency channels occupied by the primary users. Overall, cognitive radio is based on dynamic spectrum access [6] depends on the vicinity of a channel. When the channel is not used by primary users, it is a very easy task to occupy that channel for a secondary user via spectrum sensing technique but the problem occurs at the time of increased traffic of primary users occupy the spectrum.

Random sensing [7] is used to sense the spectrum in order to give the information to the secondary users to occupy the channel but in high traffic cognitive radio networks most of the time primary users are active due to which throughput of the cognitive radio network decreases via using random sensing which cause interference to the primary users.

Many researchers have done their researches in order to increase the throughput of the cognitive radio networks and also provide different techniques of spectrum sensing. Many authors have done their work on power allocation to the secondary users as more power generated via secondary users can cause interference to the primary users and less power allocation will affect secondary user in order to occupy the channel. Before sensing, a process is processed via secondary users known as prediction of the channel [8] in which the secondary user predicts that which channel is ideal to use. The problem is that once the secondary user start predicting the idealness of a channel, during every prediction it predict all the channels instead of predicting the interested ones or predicting those channels which are not predicted yet, this is due to lack of efficient algorithms and techniques for the channel prediction are available, this problem is called as stuck problem. The throughput gets affected due to stuck problem as the secondary users predict the channels repeatedly. Throughput in a wireless network is the amount of data which is transmitted or received over the channel in a limited time. A wireless channel is said to be an ideal channel if its throughput is equal to its bandwidth but it cannot be practically possible due to the factors like noise, jitters, scattering of the signal etc. The most important factor is the methodology or the algorithm used for predicting and sensing the channel as if the algorithm is more complex or time taking, the throughput of the network will be affected by these factors and if the algorithm is very simple and easy to implement then the security of the network will be compromised so there exist a need of a methodology that consider the important factors for the better performance of cognitive radio.

There are many algorithms used for spectrum sensing like ant colony sensing algorithm which is based on an idea of ant colonies in which an ant hang around for food finding and after that ant gets the food, all the ants follow the path of the previous one to get the food and the path sign are 
left by front ant by spreading a chemical from their body on that path. ACS (ant colony sensing) [9] is a very simple and efficient technique to sense the ideal channels so that secondary users or cognitive users can occupy those channels. Like ACS there is an artificial bee colony sensing technique which is based on the process followed by the bees in order to get their food finding. This technique is a robust technique and also shows better results as compared to ACS technique. Dynamic channel assignment, fixed channel assignment, frequency reuse, game theory and many more techniques have been developed in order to get an efficient methodology for channel assignment in such a way that users can use whole bandwidth assigned to the user.

A methodology is proposed in this research paper for decreasing the false alarm probability $\mathrm{P}_{\mathrm{f}}$ and increasing the sensing probability $\mathrm{P}_{\mathrm{s}}$ in order to get an efficient cognitive system. The proposed methodology is an enhanced artificial bee colony technique including a modified prediction strategy for the secondary users in order to decrease the sensing time via predicting ideal channels before sensing. Many of the researchers has done the research by focusing on only sensing of the channel but if secondary user will predict the probability of the channel to be ideal, the sensing time can automatically be improved via eliminating the occupied channels or the channels from the list which are already sensed.

Dorigo, M. et al. [10] proposed an algorithm for sensing the channel to be ideal called as an ant system. Ant system based on the daily life behavior of real ants, an ant always tends to calculate the shortest paths in order to do their food findings, all other ants wait till the front warrior ant doesn't find the food, after food finding all the ants are triggered via first ant and the whole ant colony follow the path found via first ant. Dorigo et al. added some artificial parameters like visibility, transportation method etc to the behavior of real ants in order to solve the problem of transportation in a network. Many optimizations are done in past years to ant system in order to increase the efficacy of the algorithm. This algorithm has many applications like in network routing, resource management, multi target networks, parallel arrangements etc. The main drawback of this technique is that, it is used to sense the system in sequential ordering of the ants which cannot be controlled. The ACS technique was initially based on graph coloring which was not practically adaptable for fast sensing of the channels in a network.

B. Sarala et al. [11] discussed about energy detection technique used for detection of the channel based on the comparison of the energy level of the primary users.

$$
E=\frac{1}{M} \sum_{t=1}^{M}\left|y(t)^{2}\right|
$$

After calculating the average energy of the channels, it is compared to the threshold energy $E_{t}$, the output of the energy detector can be shown as:

$$
\begin{aligned}
& \varnothing=\mathrm{H}_{1}, \text { if } \mathrm{E}>\mathrm{E}_{\mathrm{t}} \\
& \varnothing=\mathrm{H}_{0}, \text { if } \mathrm{E}<\mathrm{E}_{\mathrm{t}}
\end{aligned}
$$

The main advantage of the energy detector is the low cost to implement such a system and also this technique does not required any prior information about the primary user that is why the detection time of this technique is very low. With the advantages, this technique has some disadvantages also as the high amplitude unwanted signals affect the communication so it is 
difficult to detect the exact location of the primary user. The false alarm probability is high as compared to other techniques.

F. Koroupi et al. [12] developed later an enhanced ant colony system which is compared to the previous versions like graph coloring method etc. and prove themselves better than previous techniques. In their research paper they proposed an optimized algorithm which is an enhanced version of graph coloring problem for different numbers of primary and secondary users and also for different numbers of the channels. Researchers have stimulated their results inn MATLAB 2009 b on a desktop computer with core 2 quad 2.5 GHZ CPU and 3 GB RAM. The results are far better as compared to the results of Dorigo M. et al. [9] To increase the flexibility of the secondary users they have assigned some rate parameters which can be changed according to the requirement of the users as well as the channel. These parameters can also be changed in order to increase adaptability to the characteristics of the network. The proposed algorithm is flexible and simple to implement according to the network requirements but the drawback is that its probability distribution can be changed for each iteration that means that means the proposed algorithm has a dependent sequence of random decisions.

Li X. et al. [13] discussed about artificial bee colony algorithm with memory in their research paper, the artificial bee colony algorithm works on the basis of the behavior of the real honeybees colony. This algorithm have very less number of control parameters so that it is easy to control the flow of data and changes can be made easily according to the network requirements in order to increase the efficiency of the algorithm for sensing a channel. They have done memory synchronization with artificial bee algorithm after studying the biological behavior of the honeybees.

Artificial bee colony algorithm is a swarm based algorithm which was introduced in 2005 to overcome the drawbacks of ants colony system. The whole bee colony system consists of three main components called as unemployed bees, employed bees and the sources of food. A number of artificial bees search for their foods and propose $n$ number of solution vectors after that they continuous search for better solution in neighbor to adapt them and leave behind the poor ones. This algorithm gives better results in the means of sensing time and probability distribution. It was firstly used for only numerical optimization, after that this techniques proves itself a robust and easy implemented for maximum applications in wireless networks, neural networks, image processing etc.

The time frame for spectrum hole utilization consist of three elements that are prediction time, sensing time and transmission time. The change in any of the entity will affect the other one. Suppose if prediction time is increased by some value $\mathrm{x}$, the time for sensing or transmission will be decreased because there is a limited time period for a frame. Mohsin Ali and Haewoon Nam [14] have flourished the analytical framework to maximize the probability of spectrum hole utilization for a cognitive radio network. The proposed methodology by them is based on soft cooperative spectrum sensing. But the drawback of the proposed algorithm is that increased sensing time in a time frame reduced automatically the transmission time of the user which can results in reduced spectrum utilization.

Many of the researchers have been proposed the different techniques for spectrum prediction, Yarkan and Arslan [15] have discussed in detail about the spectrum prediction technique by using binary time series approach. It depends totally on the state of the already available 
spectrum in order to predict the next state of the spectrum. The major drawback of this technique is that the binary tine series is non-deterministic. After that this algorithm is enhanced in order to provide the prediction without having prior knowledge of the state of the spectrum. These techniques were adapted successfully by the network topologies as these algorithms resulted in decreasing false alarm probability and increased sensing probability as the secondary user does not required to predict the channel which is already predicted repeatedly.

It is observed that the interference to the primary users is very high in above discussed technique which has to be minimized as to get the maximum throughput. Cristian and Moh [16] have discussed an algorithm named as advanced low interference channel status prediction algorithm which has improved errors and interference to the primary users. By using this algorithm the interference of the primary users is effectively reduced by $40 \%$ which was far better as compared to the previously deployed algorithms, but this algorithm is not able to sense the channel, it just predict the condition of the channel.

Many algorithms are discussed above in order to find out an effective solution for the spectrum allocation for secondary users in cognitive radio. Some of the algorithms has been discussed which are sensing the allocated channel for primary users but there were no prediction of the channel due to which one channel is sensed multiple times which is the cause of less throughput of the network, some researchers has been proposed algorithm for the prediction of a channel allocated to primary user but they are not able to do effective sensing of the channel. The proposed algorithm in the proposed research is an effective solution for the drawbacks of above discussed algorithm as well as methodologies for allocation of spectrum for secondary users in cognitive radio.

\section{METHODOLOGY}

The proposed methodology in this research paper is focused on whether the prediction of the spectrum is ideal or not and also concentrated on the efficient sensing technique. The frame structure in cognitive radio system generally distributed in two parts that are sensing and transmission, both are proportional to each other as shown in the Figure 1. Firstly, the channel is sensed by secondary users by using some sensing algorithms as means of gaining the information about the channel whether it is ideal or not. After sensing the ideal channel secondary users emits the power in order to occupy the vacant bandwidth of the channel.

If the sensing time is increased, the transmission time will be decreased and vice-versa. If all the time will be wasted in sensing the channel, the throughput of the network will be decreased in accordance with the transmission time. The time taken for sensing the spectrum can be further divided in two parts, one will be used for sensing itself and the other one will be used for spectrum prediction. The prediction will help in increasing the throughput of the network via predicting the ideal channels then after sensing will be processed by which the time taken to sense all channels can be effectively reduced.

For the prediction of the spectrum a simple and effective algorithm has been introduced in this paper. The time frame for transmitting the data over the network is divided in three parts that are prediction time, sensing time and transmission time as shown in Figure 2. 
According to a theory in probability, the estimation of the probability of next event can be done by calculating the ratio of number of successful events and total number of the events to be done. So after predicting the ideal channels from say n number of channels of one or more networks, the probability of next channel to be an ideal can be find out by above explained theory. As the whole calculation is dependent of pre sensing results so stuck problem can be occurred due to which a simple algorithm has been proposed.

After predicting the channel it comes about sensing the channel and for that an enhanced honey bee colony algorithm is proposed in this research paper. The interference between a primary user and a secondary user cannot be neglected as it tends to reduce the throughput of the network. As a pair of secondary users and a primary user is considered in a network where primary user is active in its channel and the pair of the secondary users is also active in the same channel trying to use the left bandwidth within the channel is shown in the Figure 3.

When a secondary user wants to use the channel in which a primary user is active, there is a chance of mutual interference occurrence between primary and secondary user. It occurs when secondary user transmit more power in order to use the channel as shown in the Figure 3. There are two secondary users SUx and Suy interfere the channel allocated to PU which is a primary user. R1 is the radius of the cell generated via a secondary user by using more power than required, Rt is the radius of the cell which should be generated via a secondary user in order to eliminate the chances of the mutual interference.

However, the mutual interference can be eliminated via controlling the power transmitted by the secondary users. Matric $\mathrm{M}$ is formed of the size $\mathrm{S} * \mathrm{C}$ where $\mathrm{S}$ is the number of the secondary user and $\mathrm{C}$ is the number of the channel. It is obvious that all the channels are not available for use of the secondary users so the value of some $\mathrm{M}_{\mathrm{sc}}$ is 1 which shows that the secondary user can use this channel.

$$
M=\left[\begin{array}{lllllll}
1 & 0 & 1 & 0 & 0 & 0 & 1 \\
0 & 1 & 0 & 1 & 0 & 0 & 0 \\
0 & 0 & 1 & 0 & 1 & 0 & 0 \\
0 & 0 & 0 & 1 & 0 & 0 & 0 \\
1 & 0 & 1 & 0 & 1 & 0 & 0
\end{array}\right]
$$

$\mathrm{M}_{\mathrm{xy}}$ is a channel availability matrix.

$$
A=\left[\begin{array}{lllllll}
1 & 0 & 0 & 0 & 0 & 0 & 1 \\
0 & 1 & 0 & 0 & 0 & 0 & 0 \\
0 & 0 & 1 & 0 & 1 & 0 & 0 \\
0 & 0 & 0 & 1 & 0 & 0 & 0 \\
1 & 0 & 0 & 0 & 1 & 0 & 0
\end{array}\right]
$$

$\mathrm{A}_{\mathrm{xy}}$ is spectrum assignment matrix.

$$
E=\left[\begin{array}{llllllllllllll}
1 & 0 & 0 & 1 & 1 & 0 & 0 & 1 & 1 & 0 & 1 & 0 & 1 & 0
\end{array}\right]
$$

$E_{x y}$ is the encoded method. 
A pool of $n$ number of solution is taken from the channel matrix $M$ in order to find out the fitness value of the solutions. The pool is shown in the matrix A. from the matrix A the encoded solution can be generated via a recognized encoded method.

If the state of the primary user is ideal, the throughput of the secondary user will be given as

$$
\mathrm{X}_{0}=\frac{T-\tau p-\tau s}{T} \log 2(1+S N R s)
$$

And if the state of the primary user is active the throughput to the secondary user will be given as

$$
\mathrm{X}_{0}=\frac{T-\tau p-\tau s}{T} \log 2\left(1+\frac{S N R s}{1+S N R p}\right)
$$

Where,

- $\mathrm{T}$ is the total time taken for transmission

- $\quad \mathrm{C}_{\mathrm{p}}$ is threshold for primary user

- $r_{s}$ is threshold for secondary user

- $\mathrm{SNR}_{\mathrm{s}}$ is signal to noise ratio for secondary user

- And, $\mathrm{SNR}_{\mathrm{p}}$ is signal to noise ratio or primary user.

Initially, the random selection was used for prediction of the channels, the secondary user randomly predict the channel for sensing and starts communication if the channel is ideal, but if the sensed channel is not an ideal then again secondary user starts predicting the channel randomly and it is the probability that secondary user can predict the same channel again. So the random technique was unsuccessful in order to predict the channel effectively. The proposed methodology allows secondary users to calculate the probabilities of the channels to be ideal. First the secondary user will calculate the probability of a pool of the channels and will take concern for the probabilities more than 0.5 , if a channel has less probability to be ideal than 0.5 , the secondary user will leave it from the selected pool. Now, after calculating the probabilities of the channels secondary user will arrange them in decreasing order so that, the channel of the higher probability can be sensed first.

Figure 4 illustrates the principle steps of the proposed algorithm wherein the enhanced $A B C$ calculation is propelled by the drawbacks of the past work done. The initial step is introduction, in which various beginning arrangements (n) are created by means of the encoding strategy for a given matrix M. The wellness estimations of these arrangements are assessed advanced model. The channels of high probability prediction are determined from the selected colonies in order to get the best results. The selected pool of the solutions is mutated with the new pool in order to select the best results among both pools of the solutions. The pool matrix is generated via selecting best results from the channel availability matrix and after that secondary user is ready to do communication via selected channel with best probability to be ideal. If the process is not terminated, it will repeat itself till the channel is not ready for communication between secondary transmitter and receiver. Hence, the channel with highest ideal predicted probability is sensed and ready for doing communication via secondary user. However, the proposed methodology eliminates the problem of sensing a channel repeatedly and the proposed method has better output in the terms of its throughput, noise interaction etc. 


\section{RESULTS AND DISCUSSIONS}

An intel dualcore 2.6 GHZ CPU is used for the stimulation of the proposed methodology on the software MATLAB 2017a. To get the results here a CRN network area of square dimensions are considered. The network area consist both primary and secondary users whereas the primary users transmitting via a base station installed in network area. Primary users have their transreceiver in order to perform the communication and the secondary users have the transmitters and receivers individually in order to maintain some distance between transmitters and receivers for travelling of the transmitted signal. The sensing is performed at 50 instances in the cognitive radio network in order to get the value of the parameters at different instances for comparing them with the parameters obtained via performing binary artificial bee colony algorithm on the same network of same dimensions in order to prove the efficiency of the proposed methodology.

The proposed methodology is able to optimize the network utilization as it produce high spectrum usage efficiency. As the number of secondary users will be increased, the spectrum usage efficiency will also be increased. The graph in Figure 5 shows the efficiency for 50 CRN instances. The proposed method is robust as compared to binary artificial bee colony algorithm and according to the obtained results the proposed enhanced artificial bee colony algorithm shows $11.48 \%$ improvement in the terms of spectral usage efficiency of the cognitive radio network.

Binary artificial algorithm shows good results and it is an efficient technique in order to solve the tree problem that occurs in a network but after comparing the stimulation parameters of BABC and the proposed methodology it is proved that the proposed methodology has better results in terms of median, standard deviation and spectrum usage efficiency. The number of solutions for performing $\mathrm{BABC}$ and the proposed methodology are taken 20 and the limit is taken $\mathrm{n}^{*} 0.5^{*} \mathrm{D}$. The crossover operation is applied to each and every solution. The spectrum usage efficiency is shown in the Figure 5 in which the brown line in the graph represents the spectrum usage efficiency obtained by performing the proposed methodology and the yellow line represents the spectrum usage efficiency obtained via performing binary artificial bee colony algorithm.

The Figure 6 shows the dependency of spectral usage efficiency on the number of solutions. $\mathrm{n}$ is only the parameter which can be fluctuate according to the requirement. Here the value of $n$ are taken is 70 and the spectrum usage efficiency is measured at 7 instances where the value of $\mathrm{n}$ are 10, 20, 30, 40, 50, 60 and 70. It can be seen clearly in the Figure 6 that the value of spectrum usage efficiency gradually increases as the number of solution increase. It is minimum when the value of $\mathrm{n}$ is 10 and after some time it got constant at the maximum amplitude.

\section{CONCLUSION}

The demand of wireless communication is sky high now days. To fulfill the demand of the users via using available frequency spectrum for all worldwide users, it is very difficult task to provide a single channel for the communication to every user. So there comes the need to use the available spectrum efficiently and for that the cognitive radio networks were developed which is best solution for the problem of spectrum usage. There are multiple methodologies or algorithm used for spectrum allocation to the secondary users and to increase the spectral usage efficiency 
in order to provide an optimum solution to the secondary users but the throughput of the cognitive radio network should also be in mind while implementing such algorithms because the interference introduced to the channel allocated to primary users tends to decrease the throughput of the network.

The proposed methodology in this research paper optimized the spectrum usage efficiency that means it enhance the usage of the available spectrum between primary and secondary users without interfering the active communication path of the primary user. The stuck problem is also solved by using enhanced artificial bee colony algorithm. The results show that the proposed algorithm is $11.48 \%$ more efficient in the terms of better spectrum usage efficiency as compared to the binary artificial bee algorithm. It is found from the Figure 6 that the value of number of solutions should be set to 40 in order to get the best results however, the value more than 40 has very less effect on the value of spectrum usage efficiency.

\section{REFERENCES:}

[1] O. Ur-Rehman and N. Zivic, "Wireless communications," in Signals and Communication Technology, 2018.

[2] R. Ling, "Mobile Communication," in International Encyclopedia of the Social \& Behavioral Sciences: Second Edition, 2015.

[3] A. Goldsmith, "Path Loss and Shadowing," in Wireless Communications, 2013.

[4] P. J. Pupalaikis, "Scattering Parameters," in S-Parameters for Signal Integrity, 2020.

[5] S. Haykin, "Cognitive radio: Brain-empowered wireless communications," IEEE J. Sel. Areas Commun., 2005, doi: 10.1109/JSAC.2004.839380.

[6] E. Hossain, D. Niyato, and Z. Han, Dynamic spectrum access and management in cognitive radio networks. 2009.

[7] Y. Zeng, Y. C. Liang, A. T. Hoang, and R. Zhang, "A review on spectrumsensing for cognitive radio: Challenges and solutions," Eurasip Journal on Advances in Signal Processing. 2010, doi: 10.1155/2010/381465.

[8] V. K. Tumuluru, P. Wang, and D. Niyato, "Channel status prediction for cognitive radio networks," Wirel. Commun. Mob. Comput., 2012, doi: 10.1002/wcm.1017.

[9] M. Dorigo and T. Stützle, "Ant colony optimization: Overview and recent advances," in International Series in Operations Research and Management Science, 2019.

[10] S. H. Chia, K. L. Su, J. H. Guo, and C. Y. Chung, "Ant colony system based mobile robot path planning," 2010, doi: 10.1109/ICGEC.2010.59.

[11] J. J. J. S. a B. Sarala a,*, S. Rukmani Devi b, "Spectrum energy detection in cognitive radio networks based on a novel adaptive threshold energy detection method," Comput. Commun., vol. 152, pp. 1-7, 2020.

[12] F. Koroupi, S. Talebi, and H. Salehinejad, "Cognitive radio networks spectrum allocation: 
An ACS perspective,” Sci. Iran., 2012, doi: 10.1016/j.scient.2011.04.029.

[13] X. Li and M. Yin, "A discrete artificial bee colony algorithm with composite mutation strategies for permutation flow shop scheduling problem," Sci. Iran., 2012, doi: 10.1016/j.scient.2012.10.034.

[14] M. Ali and H. Nam, "Optimization of spectrum utilization in cooperative spectrum sensing," Sensors (Switzerland), 2019, doi: 10.3390/s19081922.

[15] S. Yarkan and H. Arslan, "Binary time series approach to spectrum prediction for cognitive radio," 2007, doi: 10.1109/VETECF.2007.332.

[16] I. Christian and S. Moh, "A Low-Interference Channel Status Prediction Algorithm for Instantaneous Spectrum Access in Cognitive Radio Networks," Wirel. Pers. Commun., 2015, doi: 10.1007/s11277-015-2922-0. 


\section{Figures}

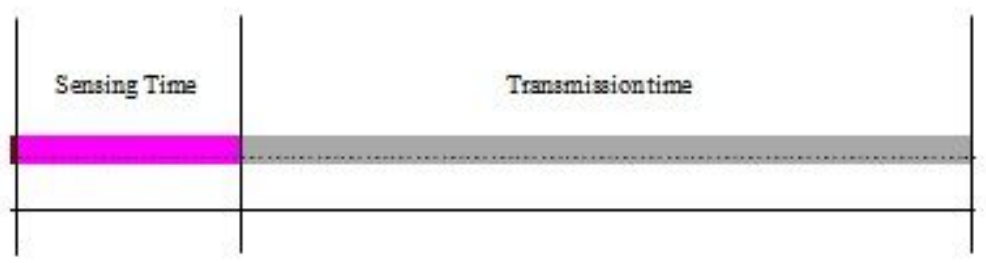

\section{Figure 1}

The traditional frame structure used in cognitive radio networks, the frame is divided in two parts. First part is used in order to perform sensing the channel and second part is used for transmission of data over the channel.

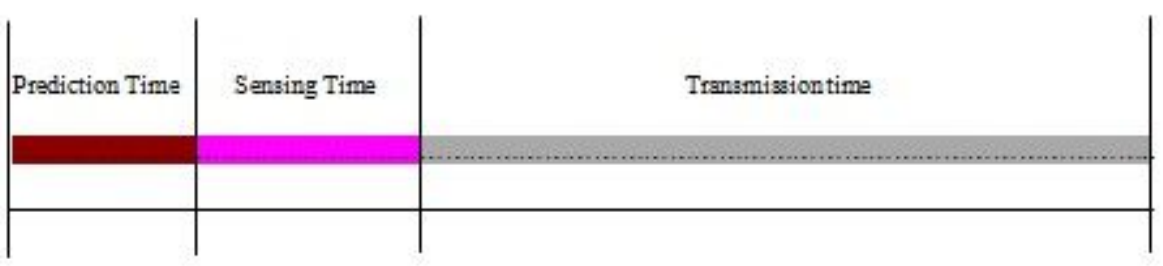

\section{Figure 2}

The frame structure of cognitive radio with splitting sensing time in two parts, one for predicting the channel and other for sensing of the channel and the rest part of the frame is used for transmission of the data.

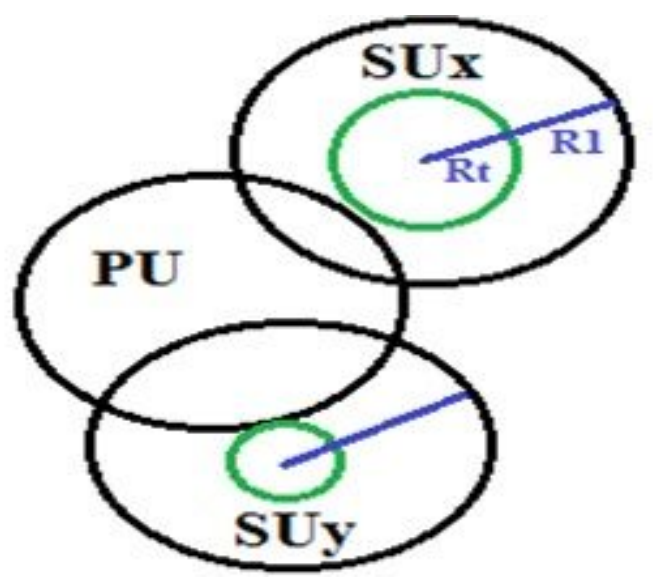

\section{Figure 3}

The interference occurs when a secondary user wants to communicate via channel allocated to the primary user, green circle shows that if secondary user will communicate up to those boundaries, primary user will not be affected. 


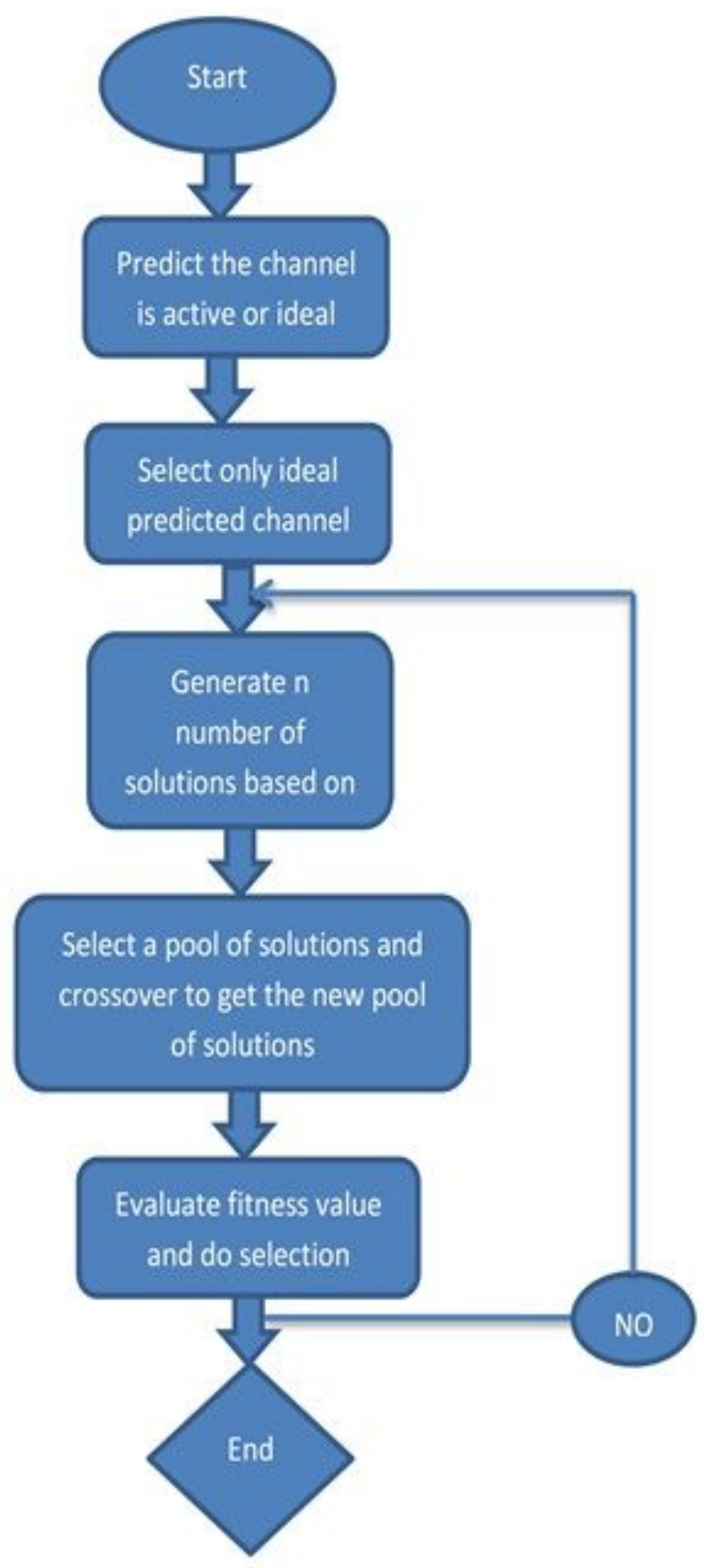

\section{Figure 4}

The proposed methodology for efficient spectrum allocation to secondary user in cognitive radio, first three steps show the method of predicting the channel and rest of steps shows the methodology adapted in order to perform sensing of the channel. 


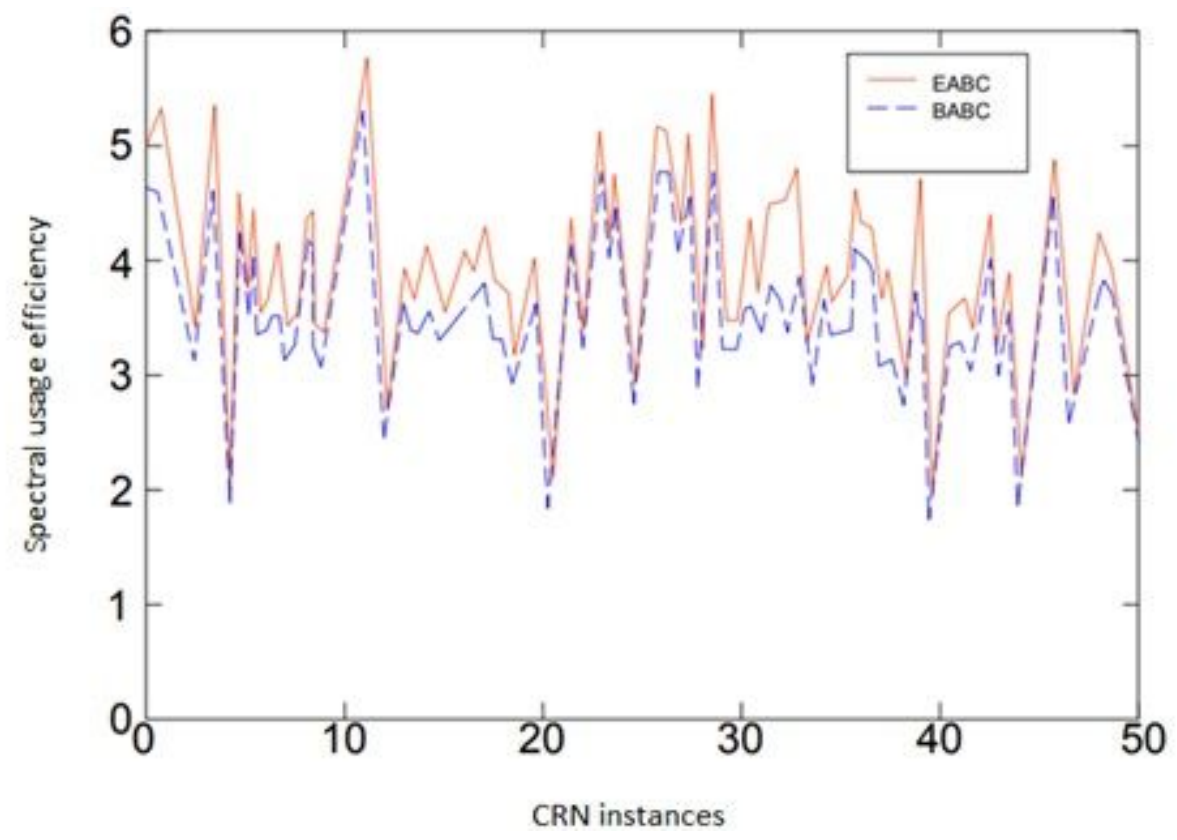

Figure 5

The comparison of spectrum usage efficiency between proposed algorithm and binary artificial bee colony algorithm. As it can be seen from the above mentioned graph that the spectrum usage efficiency of enhanced artificial bee colony algorithm is better as compared to binary artificial bee colony algorithm.

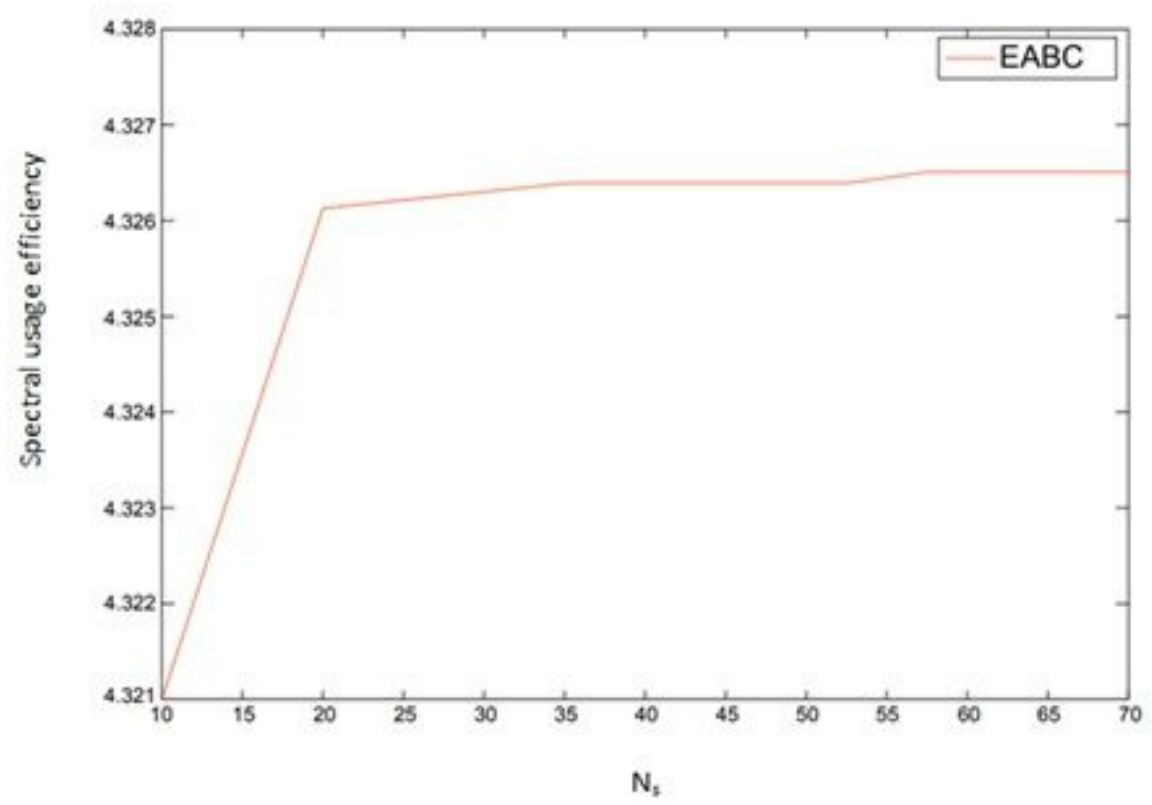

Figure 6

Spectrum user efficiency for different colony sizes as it can be seen from the graph that the spectrum usage efficiency gradually increases between the colony size of 10-20 but after 20 the line that represents spectral usage efficiency becomes constant. 